\title{
LESÕES EM TENISTAS AMADORES NO RIO DE JANEIRO
}

INJURIES IN AMATEUR TENNIS PLAYERS IN RIO DE JANEIRO

LESIONES EN TENISTAS AMATEURS EN RIO DE JANEIRO

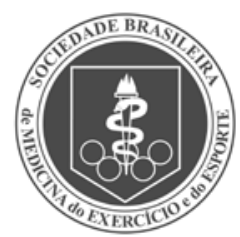

Artigo Original
Nelcimar Souza Queiroz (Educador Físico)

Patrícia dos Santos Vigário (Educadora Física)

Lilian Ramiro Felicio ${ }^{1}$ (Fisioterapeuta)

Míriam Raquel Meira Mainenti (Educadora Física)

1. Centro Universitário Augusto Motta (UNISUAM), Rio de Janeiro, RJ, Brasil.

\section{Correspondência:}

Míriam Raquel Meira Mainenti. Programa de Pós Graduação em Ciências da Reabilitação. Praça das Nações, 34, $3^{\circ}$ andar, Bonsucesso, Rio de Janeiro, Brasil, 21041-010. miriam.mainenti@hotmail.com

\section{RESUMO}

Introdução: O treinamento desportivo em diversas modalidades está associado à presença de lesões, principalmente quando apresenta grande volume e intensidade. Poucos estudos nacionais investigaram a prevalência de lesão em tenistas, porém, as informações sobre a localização, o tipo e o mecanismo das lesões que acometem esse grupo de atletas são importantes para a prevenção e o tratamento de tais lesões. Objetivo: Verificar a frequência de lesão relatada por tenistas amadores no município do Rio de Janeiro. Métodos: A avaliação foi realizada por meio de um questionário estruturado, com informações sobre a região corporal acometida, tipo e mecanismo de lesão, além de dados sobre a característica do treinamento. Os grupos formados, lesão e não lesão, foram comparados pelo teste $t$ de Student ou Mann-Whitney e pelo teste Qui-quadrado ou Exato de Fisher, no programa SPSS ( $p<0,05)$. Resultados: Dos 159 jogadores de tênis $(89,3 \%$ homens; $45,3 \pm 11,4$ anos de idade; $8,5 \pm 6,6$ anos de prática esportiva) que responderam o questionário, 38,4\% relataram já ter sido acometidos por alguma lesão decorrente do tênis. As regiões mais acometidas foram: cotovelo $(24,5 \%)$, joelho $(11,3 \%)$ e tornozelo $(6,9 \%)$. Considerando todas as lesões, a tendinite foi o tipo mais relatado (24,5\%), seguido por entorse $(12,6 \%)$ e lesão meniscal $(4,4 \%)$. Com relação ao mecanismo de lesão, os mais prevalentes foram por repetição $(25,2 \%)$ e mudança de direção $(15,1 \%)$. O grupo lesão apresentou maiores valores para idade ( $48,8 \pm 10,1$ vs. $43,6 \pm 11,8$ anos), tempo de prática da modalidade (10,5 $\pm 8,5$ vs. $7,2 \pm 4,8$ anos) e massa corporal total $(86,8 \pm 9,8$ vs. $81,0 \pm 11,0 \mathrm{~kg})$. Conclusão: A prevalência de lesão em tenistas amadores no Rio de Janeiro foi de $38,4 \%$, sendo a epicondilite lateral do cotovelo a mais frequente, em virtude da repetição do gesto esportivo.

Palavras-chave: traumatismos em atletas, prevalência, cotovelo de tenista, educação física e treinamento.

\section{ABSTRACT}

Introduction: Sports training in various modalities is associated with injuries, especially when presenting large volume and intensity. Few Brazilian studies have investigated the prevalence of injury in tennis players, however, information about the location, type and mechanisms injuries which affect this group of athletes are important for prevention and treatment of such injuries. Objective: To determine the frequency of lesions reported by amateur tennis players in the city of Rio de Janeiro. Methods: The evaluation was conducted through a structured questionnaire with information about the affected body region, type and mechanism of injury, as well as data on the characteristics of the training. The groups formed, injury and non injury, were compared by Student's t test or Mann-Whitney test, and the chi-squared or Fisher's exact test, using SPSS software ( $p<0.05)$. Results: Of 159 tennis players ( $89.3 \%$ men, $45.3 \pm 11.4$ years old; $8.5 \pm 6.6$ years of tennis practice) which completed the questionnaire, $38.4 \%$ reported having been affected by an injury arising from tennis. The most affected regions were: elbow (24.5\%), knee (11.3\%), and ankle (6.9\%). Considering all injuries, tendinitis was the most frequently reported (24.5\%), followed by sprain (12.6\%) and meniscal injuries (4.4\%). Regarding the mechanism of injury, the most prevalent were repetition (25.2\%) and changes of direction (15.1\%). The injury group presented higher age (48.8 $\pm 10.1 \mathrm{vs.} 43.6 \pm 11.8$ years), years of tennis practice (10.5 \pm 8.5 vs. $7.2 \pm 4.8)$, and total body mass $(86.8 \pm 9.8 \mathrm{vs} .81 .0 \pm 11.0 \mathrm{~kg})$. Conclusion: The prevalence of injuries in amateur tennis players in Rio de Janeiro was 38.4\%, being lateral epicondylitis of the elbow the most frequent, due to the repetition of the characteristic motor actions of this sport.

Keywords: athletic injuries, prevalence, tennis elbow, physical education and training.

\section{RESUMEN}

Introducción: El entrenamiento deportivo en diversas modalidades está asociado a la presencia de lesiones, principalmente cuando presenta gran volumen e intensidad. Pocos estudios nacionales investigaron la prevalencia de lesión en tenistas, no obstante, las informaciones sobre la localización, el tipo y el mecanismo de las lesiones que acometen a ese grupo de atletas son importantes para la prevención y el tratamiento de tales lesiones. Objetivo: Verificar la frecuencia de lesión relatada por tenistas amateurs en el municipio de Rio de Janeiro. Métodos: La evaluación fue realizada por medio de un cuestionario estructurado, con informaciones sobre la región corporal acometida, tipo y mecanismo de lesión, además de datos sobre la característica del entrenamiento. Los grupos formados, lesión y no lesión, fueron comparados por el test t de Student o Mann-Whitney y por el test del Chi-cuadrado o Exacto de Fisher, en el programa SPSS ( $p<0,05)$. Resultados: De los 159 practicantes de tenis $(89,3 \%$ hombres; $45,3 \pm 11,4$ años de edad; $8,5 \pm 6,6$ años de práctica deportiva) que respondieron el cuestionario, 38,4\% relataron ya 
haber sido acometidos por alguna lesión proveniente del tenis. Las regiones más acometidas fueron: codo (24,5\%), rodilla (11,3\%) y tobillo (6,9\%). Considerando todas las lesiones, la tendinitis fue el tipo más relatado (24,5\%), seguido por entorsis $(12,6 \%)$ y lesión meniscal $(4,4 \%)$. Con relación al mecanismo de lesión, los más prevalentes fueron por repetición $(25,2 \%)$ y cambio de dirección $(15,1 \%)$. El grupo lesión presentó mayores valores para edad $(48,8 \pm 10,1 \mathrm{vs}$. $43,6 \pm 11,8$ años), tiempo de práctica de la modalidad (10,5 $\pm 8,5 \mathrm{vs} .7,2 \pm 4,8$ años) y masa corporal total (86,8 $\pm 9,8$ vs. 81,0 $\pm 11,0 \mathrm{~kg}$ ). Conclusión: La prevalencia de lesión en tenistas amateurs en Rio de Janeiro fue de 38,4\%, siendo la epicondilitis lateral del codo la más frecuente, en virtud de la repetición del gesto deportivo.

Palabras clave: traumatismos en atletas, prevalencia, codo de tenista, educación física y entrenamiento.

\section{INTRODUÇÃO}

A prática do tênis no Brasil teve início em 1898', contudo o esporte começou a se tornar popular apenas na última década de 90, quando o tenista Gustavo Kuerten (Guga) conquistou, pela primeira vez, o campeonato de Roland Garros ${ }^{2}$. Essa conquista brasileira inédita estimulou uma maior participação de diferentes grupos na modalidade, incluindo crianças, jovens e adultos, com objetivos diversos, desde a promoção da saúde até participação e conquista de torneios esportivos.

Com o aumento no volume e na intensidade dos treinos, jogos e torneios, começam a surgir lesões, sendo as regiões mais frequentemente relatadas na literatura: cotovelo, ombro, joelho, perna, coxa, punho e coluna ${ }^{3-6}$. A epicondilite lateral, ou "cotovelo de tenista", é a inflamação do tendão, no extensor radial, longo e curto do carpo, sendo geralmente ocasionado pelo repetido atraso do backhand. Seu tratamento não depende somente dos métodos tradicionais, como os medicamentos e a fisioterapia. É de suma importância a inclusão do profissional de Educação Física, de modo a permitir a correção das técnicas dos movimentos'.

Alguns pesquisadores nacionais já verificaram a prevalência de lesões nesse grupo de atletas, como Guedes et al. ${ }^{5}$, em Santa Catarina e no Rio Grande do Sul; Keller et al. ${ }^{6}$ em Santa Catarina e Paraná; e Silva et al. ${ }^{3}$, em São Paulo. Forti e Pereira ${ }^{4}$ foram os únicos a investigar essa questão no Rio de Janeiro, porém sua amostra continha 30 indivíduos apenas, o que não representa, atualmente, o quantitativo de praticantes de tênis nessa cidade.

O conhecimento das articulações mais acometidas, bem como os tipos e mecanismos de lesão mais frequentes podem contribuir para a tomada de decisão quanto às condutas voltadas à prevenção e/ou reincidências de lesões. Objetivo do presente estudo foi verificar a frequência de lesão relatada por praticantes amadores de tênis no município do Rio de Janeiro, RJ, Brasil. Adicionalmente, buscou-se identificar que características demográficas e de treinamento estariam associados à ocorrência de lesão. A hipótese desse trabalho é que o cotovelo seja a articulação mais acometida e que a tendinite seja o tipo de lesão mais frequente, configurando o "cotovelo de tenista".

\section{MATERIAIS E MÉTODOS}

Esse foi um estudo seccional realizado pelo Laboratório de Análise do Movimento Humano (LAMH) do Centro Universitário Augusto Motta (UNISUAM), cujo protocolo foi aprovado pelo Comitê de Ética em Pesquisa Institucional (CAAE: 23954213.8.0000.5235). A primeira fase do estudo foi verificar quais eram os clubes e condomínios no município do Rio de Janeiro, RJ, Brasil, nos quais eram oferecidas aulas sistemáticas de tênis. A partir de um contato telefônico feito com a Confederação Brasileira de Tênis (CBT) foram identificadas 13 instituições na zona oeste, três na zona norte e três na zona sul, totalizando 19 clubes e/ou condomínios. A partir dessa verificação, cada instituição foi consultada e naquelas nas quais a autorização foi concedida, os alunos foram convidados a participar da pesquisa.

A pesquisa foi realizada com atletas amadores, homens e mulheres maiores de 18 anos, que jogavam tênis há mais de 12 meses e que treinavam com orientação profissional por mais de uma hora por semana. Qualquer incapacidade em compreender o questionário e a não assinatura do Consentimento Livre e Esclarecido foram determinados como critérios de exclusão.

A avaliação foi realizada através de um questionário estruturado preenchido durante uma entrevista. O questionário de prevalência de lesões foi adaptado de Brooks et al. ${ }^{8}$ e Costa ${ }^{9}$, e continha informações sobre a região corporal acometida, tipo e mecanismo de lesão, relacionados com a prática do tênis. Além disso, o questionário também continha perguntas relacionadas às características do treinamento de tênis, como duração das aulas, frequência semanal, anos de prática do esporte, participação em outro tipo de exercício/esporte e número de campeonatos disputados por ano. Foram coletados os dados de massa corporal total e estatura por auto relato.

Para investigar que fatores estavam associados à presença de lesão, os atletas foram divididos em dois grupos: lesão (pelo menos um relato de lesão) e não lesão (nenhum relato de lesão). Os dados foram apresentados como média \pm desvio padrão para as variáveis numéricas, e frequência absoluta (n) e relativa (\%) para as categóricas. A distribuição das variáveis foi verificada através do teste de Kolmogorov-Smirnov. As comparações entre os grupos do estudo foram feitas utilizando o teste tStudent (dados com distribuição paramétrica) ou Mann-Whitney (dados com distribuição não paramétrica) para as variáveis numéricas e o teste Qui-quadrado ou Exato de Fisher) para as variáveis categóricas. A análise estatística foi realizada no programa SPSS versão 13.0 para Windows (Chicago, LL, USA), sendo consideradas significativas as análises com $p<0,05$.

\section{RESULTADOS}

Doze instituições autorizaram a participação nessa pesquisa, sendo seis da zona oeste, três da zona norte e duas da zona sul da cidade do Rio de Janeiro. Foram entrevistados 159 jogadores de tênis, sendo $89,3 \%$ homens, com 8,5 $\pm 6,6$ anos de prática da modalidade. A maior parte dos entrevistados praticava tênis em turmas de nível avançado, duas vezes por semana. Aproximadamente $30 \%$ praticava outras modalidades esportivas, sendo o futebol a mais prevalente. Em relação à quantidade de campeonatos, cerca de 50\% dos entrevistados relataram participar de 1 a 4 eventos por ano (tabela 1).

Dados apresentados como média \pm desvio padrão (DP) para as variáveis numéricas e frequência absoluta (frequência relativa) para as categóricas * $p<0,05$ (teste T-Student); ${ }^{\dagger} p<0,05$ (teste Mann-Whitney).

Um total de 61 (38,4\%) participantes relatou já ter sido acometido por alguma lesão desde o início da prática desse esporte. Desses, $82,0 \%$ relataram uma lesão, $13,1 \%$ relataram duas lesões e 4,9\% relataram três lesões. $O$ local relatado mais prevalente foi o cotovelo $(n=39,24,5 \%)$, seguido pelo joelho ( $n=18,11,3 \%$ ) e pelo tornozelo ( $n=11,6,9 \%$ ) (figura 1). Agrupando as lesões, $25,2 \%$ dos atletas relataram lesão em alguma região do membro superior e $18,9 \%$ no membro inferior. 
Tabela 1. Características demográficas e de treinamento dos atletas.

\begin{tabular}{|c|c|c|c|}
\hline Variáveis & $\begin{array}{c}\text { Total } \\
(n=159)\end{array}$ & $\begin{array}{l}\text { Lesão } \\
(n=61)\end{array}$ & $\begin{array}{l}\text { Não lesão } \\
(n=98)\end{array}$ \\
\hline Homens - n (\%) & $142(89,3)$ & $58(95,1)$ & $84(85,7)$ \\
\hline Idade (anos) & $45,3 \pm 11,4$ & $48,8 \pm 10,1$ & $43,6 \pm 11,8^{*}$ \\
\hline $\begin{array}{c}\text { Massa Corporal Total } \\
(\mathrm{kg})\end{array}$ & $83,2 \pm 10,9$ & $86,8 \pm 9,8$ & $81,0 \pm 11,0^{*}$ \\
\hline Estatura (cm) & $1,79 \pm 0,11$ & $1,79 \pm 0,10$ & $1,79 \pm 0,10$ \\
\hline Tempo de Tênis (anos) & $8,5 \pm 6,6$ & $10,5 \pm 8,5$ & $7,2 \pm 4,8+$ \\
\hline \multicolumn{4}{|l|}{$\begin{array}{c}\text { Estágio de } \\
\text { Treinamento - } \mathbf{n}(\%)\end{array}$} \\
\hline Iniciante & $52(32,7)$ & $15(24,6)$ & $37(37,8)$ \\
\hline Intermediário & $40(25,2)$ & $14(23,0)$ & $26(26,5)$ \\
\hline Avançado & $67(42,1)$ & $32(52,5)$ & $35(35,7)$ \\
\hline \multicolumn{4}{|l|}{$\begin{array}{c}\text { Frequência } \\
\text { semanal - n (\%) }\end{array}$} \\
\hline 1 & $13(8,2)$ & $5(8,2)$ & $8(8,2)$ \\
\hline 2 & $78(49,1)$ & $31(50,8)$ & $47(48,0)$ \\
\hline 3 & $51(32,1)$ & $16(26,2)$ & $35(35,7)$ \\
\hline 4 & $16(10,1)$ & $8(13,1)$ & $8(8,2)$ \\
\hline 5 & $1(0,6)$ & $1(1,6)$ & $0(0,0)$ \\
\hline $\begin{array}{l}\text { Participação outro } \\
\text { exercício - n (\%) }\end{array}$ & $48(30,2)$ & $24(39,3)$ & $24(24,7)$ \\
\hline Futebol & $26(16,4)$ & $14(23,0)$ & $12(12,2)$ \\
\hline Futevôlei & $6(3,8)$ & $3(4,9)$ & $3(3,1)$ \\
\hline Vôlei de praia & $3(1,9)$ & $0(0,0)$ & $3(3,1)$ \\
\hline Natação & $3(1,9)$ & $1(1,6)$ & $2(2,0)$ \\
\hline Corrida & $3(1,9)$ & $2(3,3)$ & $1(1,0)$ \\
\hline Outros & $7(4,4)$ & $4(6,6)$ & $3(3,1)$ \\
\hline \multicolumn{4}{|l|}{$\begin{array}{c}\text { Campeonatos por } \\
\text { ano-n (\%) }\end{array}$} \\
\hline Nenhum & $18(11,3)$ & $5(8,2)$ & $13(13,3)$ \\
\hline 1 a 4 & $75(47,2)$ & $32(52,5)$ & $43(43,9)$ \\
\hline 5 a 8 & $46(28,9)$ & $16(26,2)$ & $30(30,6)$ \\
\hline Acima de 8 & $20(12,6)$ & $8(13,1)$ & $12(12,2)$ \\
\hline
\end{tabular}

Dados apresentados como média \pm desvio padrão (DP) para as variáveis numéricas e frequência absolut (frequência relativa) para as categóricas* $p<0,05$ (teste T-Student); $\uparrow p<0,05$ (teste Mann-Whitney).

A tendinite foi o tipo de lesão mais relatado, seguido por entorse e lesão meniscal. Em relação ao mecanismo de lesão, os mais prevalentes foram por repetição e mudança de direção (tabela 2).

A figura 2 apresenta os tipos de lesão relatados, considerando as regiões mais citadas: cotovelo, joelho e tornozelo. Dentre as 39 lesões de cotovelo, 38 foram tendinites e uma do tipo estiramento muscular (figura 2A). O mecanismo de lesão mais relatado para essa articulação foi a repetição do gesto $(n=38,97,4 \%)$, sendo que um único atleta $(2,6 \%)$ relatou mudança de direção como causa da lesão no cotovelo. Em relação às lesões do joelho, nove foram entorses, sete lesões meniscais, uma contusão e uma tendinite (figura 2B). Todas as 18 lesões de joelho (100\%) tiveram como mecanismo de lesão a mudança de direção. Por fim, das 11 lesões de tornozelo, 10 foram entorses e uma fratura (figura 2C), sendo 10 (90,9\%) por mudanças de direção e uma (9,1\%) por arranque.

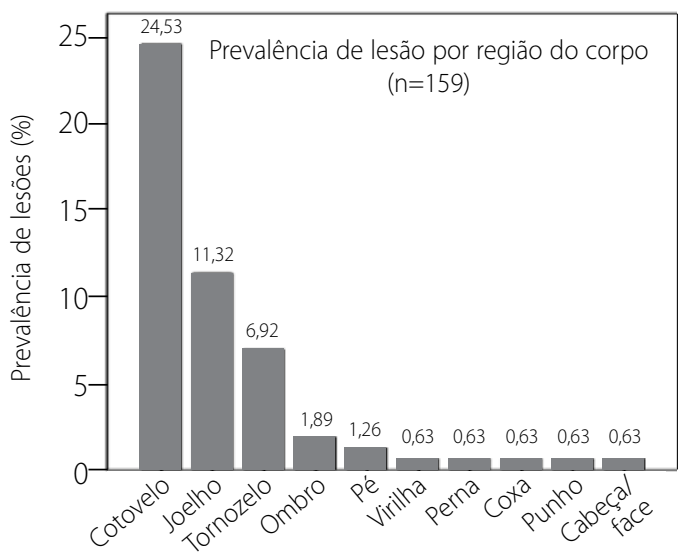

Figura 1. Prevalência de lesões segundo as regiões do corpo.

Tabela 2. Tipos e mecanismos de lesão citados pelos atletas ( $n=159)$.

\begin{tabular}{|c|c|}
\hline Tipos de lesão & n (\%) \\
\hline Tendinite & $39(24,5)$ \\
\hline Entorse & $20(12,6)$ \\
\hline Lesão meniscal & $7(4,4)$ \\
\hline Estiramento muscular & $4(2,5)$ \\
\hline Fratura & $3(1,9)$ \\
\hline Contusão & $2(1,3)$ \\
\hline Bursite & $1(0,6)$ \\
\hline Lesão ligamentar sem instabilidade & $1(0,6)$ \\
\hline Luxação/subluxação & $1(0,6)$ \\
\hline Mecanismos de lesão & n (\%) \\
\hline Repetição & $40(25,2)$ \\
\hline Mudança de direção & $24(15,1)$ \\
\hline Arranque & $2(1,3)$ \\
\hline Choque com atleta & $2(1,2)$ \\
\hline
\end{tabular}

Dados apresentados como frequência absoluta (frequência relativa).

A comparação entre os grupos lesão $(n=61)$ e não lesão $(n=98)$ mostrou que o grupo com lesão apresentava maior idade $(p=0,018)$, massa corporal total $(p=0,001)$ e tempo de prática de tênis $(p=0,002)$. Para as demais variáveis, não foram encontradas diferenças estatisticamente significativas entre os grupos (tabela 1).

\section{DISCUSSÃO}

A prevalência de lesões em tenistas amadores foi identificada em 38,4\% dos entrevistados que estavam lesionados ou já tinham sido acometidos por uma lesão. O cotovelo foi a articulação mais relatada, sendo o tipo de lesão e o mecanismo mais frequente a tendinite e o a repetição do gesto esportivo, respectivamente. Os resultados confirmaram a hipótese traçada no início da investigação sobre a presença do "cotovelo de tenista" na grande maioria dos atletas lesionados. Adicionalmente, foi identificado que o grupo lesão apresentou maiores valores para idade, tempo de prática de tênis e massa corporal total.

Os tenistas apresentam uma grande movimentação em quadra durante os jogos e treinos, com alternância de aceleração e desaceleração, e mudanças de direção para alcançar e rebater a bola. São exi- 


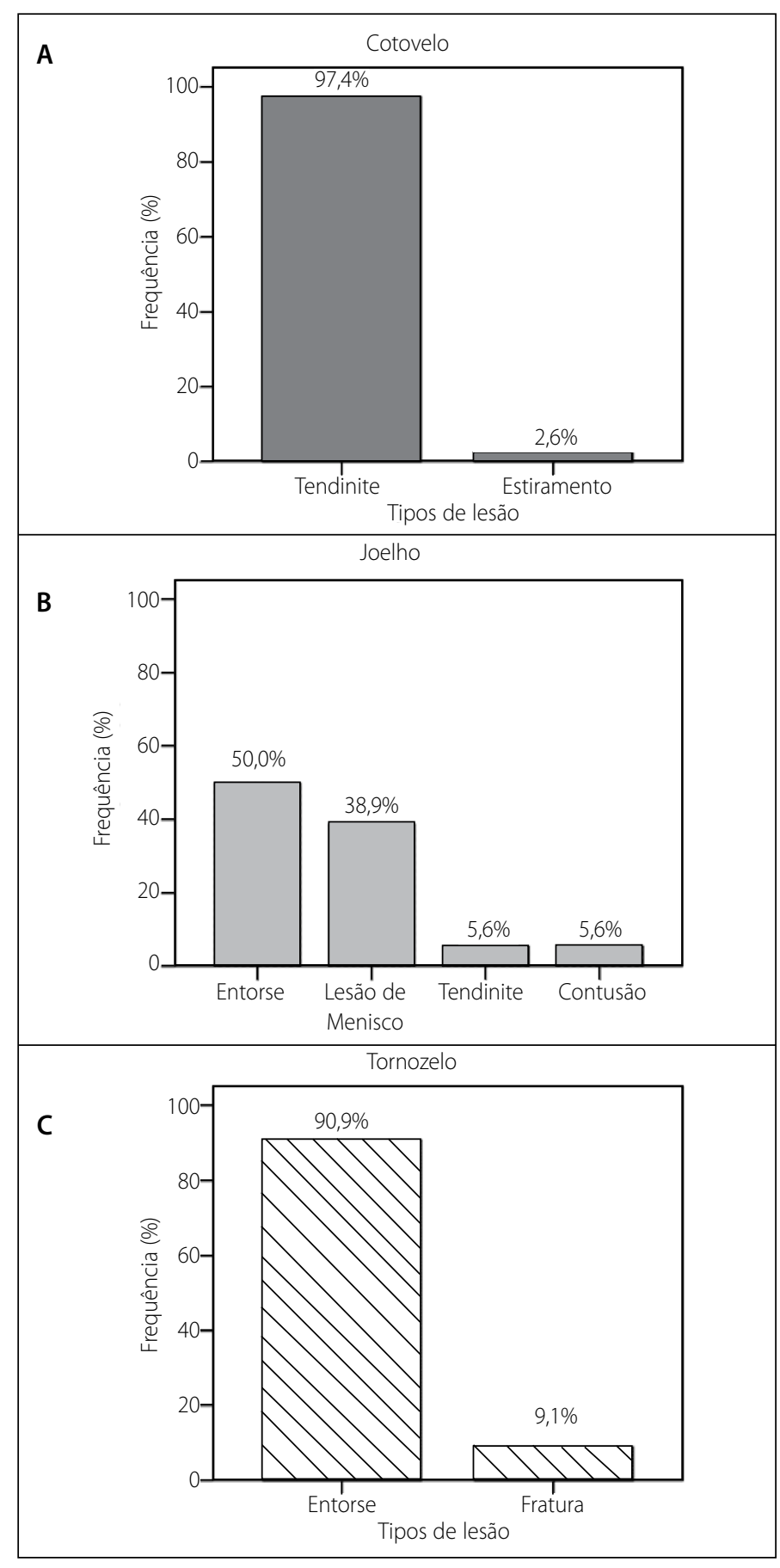

Figura 2. Prevalência de tipos de lesão segundo as regiões mais acometidas: cotovelo (A), joelho (B) e tornozelo (C).

gências de grande intensidade tanto para membros inferiores quanto nas rebatidas de bola. Fernandez-Fernandez et al. ${ }^{10}$, em uma revisão sobre o tema, mostraram que os jogadores precisam associar velocidade, agilidade e potência, com a capacidade tanto aeróbica quanto anaeróbica (intensidade de média a alta).

A repetição do gesto esportivo como fator influenciador na ocorrência de lesões e na saúde do atleta é uma grande preocupação em várias modalidades esportivas ${ }^{11-15}$, inclusive no tênis ${ }^{1,3-5,7,16,17}$.

A prevalência de lesões apresentada no presente estudo (38,4\%) foi inferior às encontradas nos estudos anteriormente realizados no Brasil: 56\% em atletas (média de 13,9 anos de prática de tênis) de Santa Catarina e do Rio Grande do Sul ${ }^{5}$, 45\% em atletas (elite da categoria infanto-juvenil) de Santa Catarina e do Paraná6, 76,2\% em atletas (média de 12,7 anos de prática de tênis) de São Paulo³, e 79,2\% em atletas amadores do Rio de Janeiro ${ }^{4}$. A menor prevalência em comparação com os demais estudos pode ter relação com o tempo de prática da modalidade, uma vez que os participantes do presente estudo apresentaram, em média, uma menor quantidade de anos de prática do tênis (8,5 anos) quando comparado aos demais estudos. Cabe ressaltar que essa variável apresentou diferença estatisticamente significativa quando foram comparados os grupos lesão e não lesão, o que revela a importância dessa variável na investigação e prevenção de lesões.

Em relação à localização da lesão, os trabalhos prévios concordam com o presente estudo, sendo o cotovelo, o ombro, o joelho e o tornozelo as regiões mais frequentemente citadas $^{3-5}$, porém apenas Guedes et al. ${ }^{5}$ corroboram os dados dessa pesquisa, quanto à região mais prevalente: o cotovelo (20,5\%).

O tipo de lesão foi estudado apenas por Forti e Pereira 4 , que encontraram maiores frequências para tendinite e entorse, como no presente estudo. Levando em consideração o mecanismo de lesão, nenhum estudo nacional discutiu esse aspecto. No presente estudo, o mais prevalente foi por repetição, tendo dois relatos de choque com atleta, resultado de jogos de duplas.

Em relação aos dados internacionais, o grupo de atletas tenistas britânicos avaliados no estudo TOYA (Training of Young Athletes Study) apresentou uma prevalência de $50 \%$ de lesão, sendo $27,8 \%$ com relato de uma e 22,2\% com relato de duas ou mais ${ }^{12}$. Em uma revisão sistemática, Pluim et al. ${ }^{16}$ mostraram que a prevalência de lesões em atletas de tênis variou de 14 a 41\%. Mais recentemente, na França, foi detectada uma prevalência de $41 \%$ de lesões no membro superior dominante dos 223 entrevistados, sendo $42 \%$ no cotovelo ${ }^{18}$.

A alta prevalência de lesão no cotovelo, do tipo tendinite, por repetição do gesto esportivo, ocorre provavelmente pelos sucessivos impactos nessa articulação, uma vez que a bola precisa ser golpeada para o campo adversário com força e velocidade ${ }^{10}$, caracterizando a potência, sem perder a precisão da rebatida, isto é, preservando a habilidade técnica para realizar o movimento. Além do impacto repetido, mudanças de direção ocorrem frequentemente, influenciando tanto os membros inferiores, quanto o membro superior que usa a raquete. A preparação física dos tenistas vem evoluindo ao longo do tempo ${ }^{19}$, permitindo um equilíbrio entre a habilidade e a capacidade de apresentá-la em situações de alta demanda energética, bastante comum nos jogos disputados por atletas de alto nível. A preparação física é importante tanto para garantir o sucesso nos jogos quanto na promoção da saúde, propondo estratégias com foco na prevenção de lesões. Um dado interessante a ser ressaltado, é que mesmo com tantos relatos de prática de outro tipo de exercício, nenhum atleta relatou participar de um programa de treinamento contra resistência, importante tanto para a prevenção, quanto para o tratamento, inclusive do "cotovelo de tenista"20,21.

Por fim, a comparação entre os grupos lesão e não lesão mostrou que aqueles indivíduos com lesão apresentavam maiores valores para idade, tempo de tênis e massa corporal total. Guedes et al. ${ }^{5}$ e Silva et al. ${ }^{3}$ corroboram o presente estudo quanto à análise da idade, pois também observaram associação entre idade e lesão. Em relação à quantidade de campeonatos por ano, Guedes et al. ${ }^{5}$ apresentaram associação com lesão, mas Silva et al. ${ }^{3}$ não encontraram, concordando com a presente pesquisa. Sobre a variável sexo, Keller et al. ${ }^{6}$ concordaram com o presente estudo e os demais estudos não realizaram essa análise.

Apesar dos interessantes resultados do presente estudo, cabe apontar a limitação relacionada à fonte da informação sobre a lesão ter sido auto relato, e não a consulta de exames e prontuários. Uma característica inerente à coleta de dados por auto relato é a possibilidade de viés de informação. Contudo, Pastre et al. ${ }^{22}$ mostraram, em uma pesquisa realizada com atletas de atletismo, que o apontamento 
do local, tipo e mecanismo de lesões apresentou alta concordância entre o inquérito de morbidade referida (relato) e o prontuário. Outra limitação foi a não inclusão dos atletas que estavam afastados por causa de lesão no período de coleta, o que pode ter subestimado a prevalência de lesão pela prática dessa modalidade.

\section{CONCLUSÃO}

A prevalência de lesão nos praticantes amadores de tênis no Rio de Janeiro foi de $38,4 \%$, sendo a principal região do corpo acometida o cotovelo, pelo tipo de lesão tendinite, em virtude da repetição do gesto esportivo. Adicionalmente, as variáveis idade, massa corporal total e tempo de prática desportiva estão associadas à ocorrência de lesões, sendo a frequência de homens e mulheres similar nos grupos lesão e não lesão.

Os profissionais que trabalham com o tênis tem um papel de grande importância, tanto na correção dos movimentos, quanto no fortalecimento muscular dos atletas, para prevenir e tratar as lesões que acometem os praticantes dessa modalidade esportiva.

Todos os autores declararam não haver qualquer potencial conflito de interesses referente a este artigo.

\section{REFERÊNCIAS}

1. Teles WA, Salve MGC. Qualidade de vida através do tênis. Mov Percepção. 2004;4(4-5):28-30.

2. Carta G, Marcher R. O Tênis no Brasil: de Maria Esther Bueno a Gustavo Kuerten. São Paulo: Conex; 2004

3. Silva RT, Cohen M, Matsumoto MH, Gracitelli GC. Avaliação das lesões ortopédicas em tenistas amadores competitivos. Rev Bras Ortop. 2005;40(5):270-9.

4. Forti D, Pereira JS. Aspectos lesionais do comprometimento osteomioarticular em praticantes amadores de tênis: estudo preliminar. Fit Perf J. 2007;6(1):53-6.

5. Guedes EJM, Barbieri DF, Fabiane F. Lesões em tenistas competitivos. Rev. Bras Cienc Esporte. 2010;31(1):217-20

6. Keller B, Braga AM, Coelho RW. Relação entre personalidade e lesões musculares em atletas de tênis de campo. Rev Bras Med Esporte. 2013;19(2):120-2.

7. Mahn PA. Uma visão do tênis de campo e suas lesões. Rev Bras Ciênc Saúde. 2004;2(4):50-3.

8. Brooks JH, Fuller CW, Kemp SP, Reddin DB. Epidemiology of injuries in English professional rugby union: part 1 match injuries. Br J Sports Med. 2005;39(10):757-66.

9. Costa MSS. Características e frequências de Lesões Musculoesqueléticas e análise estabilométrica em Bailarinos [dissertação]. Rio de Janeiro: Centro Universitário Augusto Motta; 2013.

10. Fernandez-Fernandez J, Ulbricht A, Ferrauti A. Fitness testing of tennis players: How valuable is it? $\mathrm{Br}$ J Sports Med. 2014; 48(Suppl 1):i22-31.

11. Pastre CM, Carvalho Filho G, Monteiro HL, Netto Júnior J, Padovani CR. Lesões desportivas na elite do atletismo brasileiro: estudo a partir da morbidade referida. Rev Bras Med Esporte. 2005;1 1(1):43-7.

12. Maffulli N, Baxter-Jones ADG, Grieve A. Long term sport involvement and sport injury rate in elite young athletes. Arch Dis Child. 2005;90(5):525-7.
13. Alves RN, Costa LOP, Samulsky DM. Monitoramento e prevenção do supertreinamento em atletas. Rev Bras Med Esporte. 2006;12(5):291-6.

14. Souza JMC, Faim FT, Nakashima IY, Altruda CR, Medeiros WM, Silva LR. Lesões no Karate Shotokan e no Jiu-Jitsu - Trauma Direto vs. Indireto. Rev Bras Med Esporte. 2011;17(2):107-10.

15. Sá $M C$, Victorino $A B$, Vaisberg MW. Incidência de lesão musculoesquelética sem trauma em atletas de handebol. Rev Bras Med Esporte. 2012;18(6):409-11.

16. Pluim BM, Staal JB, Windler GE, Jayanthi N. Tennis injuries: occurrence, aetiology, and prevention. $\mathrm{Br}$ J Sports Med. 2006;40(5):415-23.

17. Coelho CMO, Farias MLF, Mendonça LMC, Mello DB, Lanzillotti HS, Ribeiro BG, et al. The prevalence of disordered eating and possible health consequences in adolescent female tennis players from Rio de Janeiro, Brazil. Appetite. 2013;64:39-47.

18. Kaffel C, Creveaux T, Genevois C, Rahme M, Botta A, Rogowski I. Épidémiologie des pathologies du membre supérieur dominant chez les joueurs de tennis français. J Traumatol Sport. 2013;30(3):124-8

19. Ulbricht A, Fernandez-Fernandez J, Ferrauti A. Conception for Fitness Testing and individualized training programs in the German Tennis Federation. Sport Orthop Traumatol. 2013;29 (3):180-92.

20. Buchbinder R, Green S, Struijs P. Tennis Elbow. Clin Evid (Online). 2008;5:1117. Disponível em: http:/ www.ncbi.nlm.nih.gov/pmc/articles/PMC2907994/.

21. Peterson M, Butler S, Eriksson M, Svärdsudd K. A randomized controlled trial of exercise versus wait-list in chronic tennis elbow (lateral epicondylosis). Ups J Med Sci. 2011;116(4):269-79.

22. Pastre CM, Carvalho Filho G, Monteiro HL, Netto Júnior J, Padovani CR. Lesões desportivas no atletismo: comparação entre informações obtidas em prontuários e inquéritos de morbidade referida. Rev Bras Med Esporte. 2004;10(1):1-8. 